\title{
Rho-kinase signaling pathway promotes the expression of PARP to accelerate cardiomyocyte apoptosis in ischemia/reperfusion
}

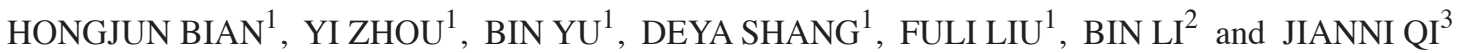 \\ ${ }^{1}$ Department of Emergency, Shandong Provincial Hospital Affiliated to Shandong University, Jinan, Shandong 250021; \\ ${ }^{2}$ Department of Health Care, Jinan Central Hospital Affiliated to Shandong University, Jinan, Shandong $250013 ;{ }^{3}$ Central \\ Laboratory, Shandong Provincial Hospital Affiliated to Shandong University, Jinan, Shandong 250021, P.R. China
}

Received July 24, 2016; Accepted May 3, 2017

DOI: $10.3892 / \mathrm{mmr} .2017 .6826$

\begin{abstract}
It has been previously reported that Rho-kinase (ROCK) and poly ADP-ribose polymerase (PARP) serve critical roles in myocardial ischemia/reperfusion (I/R) injury. Studies have additionally demonstrated that the activation of ROCK and the expression of PARP are increased in I/R. However, the effect and mechanism of the two proteins remains to be fully elucidated in I/R. In addition, whether they can be influenced by each other is unclear. In the present study, it was demonstrated that ischemia followed by reperfusion resulted in a significant increase in ROCK and PARP. In addition, Y-27632 (ROCK inhibitor) and 3-aminobenzamide (3-AB; PARP inhibitor) pretreatment rescued myocardial infarction size and cardiomyocyte apoptosis. The inhibitory role of Y-27632 was observed to be superior to that of the 3-AB group. In addition, Y-27632 and 3-AB diminished extracellular signal-related kinase (ERK) phosphorylation and the production of tumor necrosis factor $\alpha$ and interleukin 6. Overall, the results of the present study suggested that the inhibition of ROCK leads to reduced myocardial infarction size and cardiomyocyte apoptosis via the PARP/ERK signaling pathway.
\end{abstract}

\section{Introduction}

Reperfusion therapy, which ensures the return of blood supply as soon as possible to the ischemic region of the myocardium, was originally the main treatment of acute myocardial ischemia. However, Peuhkurinen (1) described reperfusion as a 'double edged sword', due to the fact that reperfusion itself can accelerate myocardial damage and lead to additional myocardial injury, which is termed ischemia/reperfusion (I/R) injury. $\mathrm{I} / \mathrm{R}$ injury can be induced by organ transplant dysfunction,

Correspondence to: Dr Jianni Qi, Central Laboratory, Shandong Provincial Hospital Affiliated to Shandong University, 324 Jingwu Road, Jinan, Shandong 250021, P.R. China

E-mail: slqijn@126.com

Key words: ROCK, PARP, ERK, ischemia/reperfusion stroke, myocardial infarction and shock, and reperfusion leads to myocardial biochemical, structural and functional changes and may determine myocardial cell survival and death (2-4). Therefore, it is important to elucidate how to improve myocardial function, reduce the arrhythmogenesis, attenuate myocardial cell apoptosis or necrosis and decrease the infarct size during I/R injury.

Rho-kinase (ROCK), a member of the serine threonine protein kinase family, is $\sim 160 \mathrm{kDa}$ in size and expressed ubiquitously in several tissues (5). ROCKs implicated in numerous types of vital movement, including the regulation of cellular contraction, growth, division, metabolism, migration, apoptosis and gene expression (6,7). According to previous studies, ROCK is closely associated with the development of a wide range of diseases including coronary heart disease, atherosclerosis, hypertension, pulmonary hypertension, heart failure, diabetes, stroke and cancer (8-10). PARP is predominantly expressed in the nucleus and can be activated under conditions including radiation, inflammation and sepsis. In the present years, accumulating data has shown that DNA strand is appear nick and breaks in I/R, at the same time, PARP is up-regulated and utilized its enzymes activation to repair these impaired DNA strands and ensure the fidelity of genomic DNA replication (11). But excessive PARP expression and activation will consumes profuse $\mathrm{NAD}^{+}$and ATP and lead to cardiomyocyte apoptosis (12). Therefore, to explore the role and relationship of ROCK and PARP in I/R will benefit us to deal with it inducing injury.

In the present study, we demonstrate that the expression of phosphorylated (p-) myosin phosphatase target (MYPT; the major effector of ROCK) and PARP are raised during I/R. In addition, 3-aminobenzamide (3-AB), which is a PARP inhibitor, is able to significantly diminish PARP expression in I/R. Notably, Y-27632, which is a ROCK inhibitor, attenuates the phosphorylation of MYPT in addition to the expression of PARP. Furthermore, these two inhibitors can rescue myocardial infarction size and cardiomyocyte apoptosis. Notably, the inhibitory role of Y-27632 was observed to be superior to 3-AB. It was verified that the mitogen-activated protein kinase (MAPK)/extracellular signal-related kinase (ERK) signaling pathway may serve a pivotal role in Y-27632 or $3-\mathrm{AB}$ protecting cardiomyocyte apoptosis induced by $\mathrm{I} / \mathrm{R}$. 


\section{Materials and methods}

Antibodies and reagents. Antibodies for phosphorylated (p-) MYPT-1 (BS4114; dilution, 1:1,500) was from Bioworld Technology, Inc. (Minneapolis, MN, USA). Antibodies for PARP (\#9542; dilution, 1:300), caspase-3 (\#9668; dilution, 1:1,000), B-cell lymphoma 2 (Bcl-2; \#2870; dilution, 1:1,000), Bcl-2-associated X protein (Bax; \#2772; dilution, 1:1,000) and p-ERK (\#9101; dilution, 1:1,000) were from Cell Signaling Technology, Inc. (Danvers, MA, USA). Antibodies for $\beta$-actin (sc-47778; dilution, 1:2,000) and horseradish peroxidase (HRP)-conjugated secondary antibodies (sc-2004 and sc-2005; dilution, 1:5,000) were purchased from Santa Cruz Biotechnology, Inc. (Santa Cruz, CA, USA). Y-27632 (ROCK inhibitor) was purchased from Calbiochem (Merck Millipore, Darmstadt, Germany). 3-AB (PARP inhibitor) was obtained from Sigma-Aldrich (Merck Millipore).

Culture and I/R of the cell line H9C2. Rat ventricular cell line H9C2 was obtained from the American Type Culture Collection (Manassas, VA, USA). The cells were cultured at $37^{\circ} \mathrm{C}$ with $5 \% \mathrm{CO}_{2}$ in DMEM containing $10 \%$ (vol/vol) FBS and $100 \mathrm{U} / \mathrm{ml}$ penicillin and $100 \mu \mathrm{g} / \mathrm{ml}$ streptomycin (Gibco; Thermo Fisher Scientific, Inc., Waltham, MA, USA). H9C2 cells were subjected to I/R. Specifically, these cells were pretreated with corresponding inhibitors and then placed into a hypoxic chamber at $37^{\circ} \mathrm{C}$ for $60 \mathrm{~min}$, then were re-oxygenated for 120 min with DMEM containing $10 \%$ FBS (13).

Hoechst staining. In order to detect myocardial apoptosis, chromatin morphology was observed with fluorescence microscopy after DNA staining with $0.5 \mathrm{mg} / 1$ Hoechst 33258 (Nanjing KeyGen Biotech. Co., Ltd., Nanjing, China). The percentage of apoptotic cells was calculated as the number of apoptotic cells compared with the number of total cells counted in 10 randomly selected fields. Images from Hoechst-stained samples were acquired by using a Leica ${ }^{\mathrm{TM}}$ fluorescence microscope equipped with a Leica ${ }^{\mathrm{TM}}$ camera (Leica Microsystems GmbH, Wetzlar, Germany).

Immunoblot analyses. For western blot analysis, cells were lysed with mammalian protein extraction reagent (CelLytic ${ }^{\mathrm{TM}}$ Cell Lysis Reagent; Sigma-Aldrich; Merck Millipore) supplemented with the protease inhibitor cocktail (\#5872; Cell Signaling Technology, Inc.). Equal amounts of protein were separated by $10 \%$ SDS-PAGE. Blots were probed with the corresponding antibodies and were incubated overnight at $4^{\circ} \mathrm{C}$. Following subsequent washing with Tris-buffered saline with Tween-20 (T1081; Beijing Solarbio Science \& Technology, Co., Ltd., Beijing, China), the membranes were incubated with the corresponding secondary antibodies conjugated with HRP for $1 \mathrm{~h}$ at room temperature. Then the membranes were visualized using a SuperSignal West Pico substrate chemiluminescence detection kit (Pierce, Rockford, IL, USA). In order to confirm uniform loading, membranes were stripped and re-incubated with anti- $\beta$-actin antibodies.

Preparation and grouping of the rat $I / R$ model. A total of 60 female Wistar rats (12-16 weeks old; body weight 250-300 g) were purchased from the Experimental Animal
Center of Shandong University (Shandong, China) and were housed individually and provided with standard lighting (12 h light and dark cycles), temperature $\left(22 \pm 0.5^{\circ} \mathrm{C}\right)$ and humidity $(60 \pm 10 \%)$ for at least 1 week prior to the experiment. All experiments were approved by the Institution Animal Care and Use Committee of Shandong Provincial Hospital Affiliated to Shandong University and were performed in line with the National Institutes of Health Guidelines. Female Wistar rats were anesthetized with urethane $(25 \mathrm{mg} / \mathrm{kg}$; intraperitoneal injection). The left thoracotomy was made in the fourth intercostal space. Subsequent to occlusion of the left anterior branch of the descending coronary artery (LAD) for $60 \mathrm{~min}$, the ligation was loosened for $120 \mathrm{~min}$. Following this, these rats were sacrificed and their hearts were obtained for the following experiments. The control rats underwent this procedure, however the LAD was not ligated. As presented in Fig. 1A, the rats were divided randomly into four groups: i) Sham operation $(n=15)$; ii) after administration of dimethyl sulfoxide (DMSO), LAD was occluded for $60 \mathrm{~min}$ and reperfused for $120 \mathrm{~min}(\mathrm{n}=15)$; iii) after administration of Y-27632 (inhibitor of ROCK), LAD was occluded for $60 \mathrm{~min}$ and reperfused for $120 \mathrm{~min}(\mathrm{n}=15)$; iv) after administration of 3-AB (inhibitor of PARP), LAD was occluded for $60 \mathrm{~min}$ and reperfused for $120 \min (n=15)$.

Evaluation of myocardial infarct size and area at risk. Following I/R, the LAD was reoccluded and $3 \mathrm{ml}$ Evans blue was injected into the left ventricle to delineate myocardial area at risk. Ischemic area (also termed area at risk, AAR) was not stained and the nonischemic area was stained blue. Subsequently, the heart was obtained and washed with normal saline. The great vessels, right ventricle and atria were removed. The tissue was frozen in a $-20^{\circ} \mathrm{C}$ freezer for $30 \mathrm{~min}$ in order to facilitate slicing. After this, the left ventricle was separated and cut into slices approximately $1 \mathrm{~mm}$ in thickness. The AAR was isolated from the area not at risk and then was incubated with nitro blue tetrazolium (NBT) solution $(1 \% \mathrm{w} / \mathrm{v})$ to distinguish between the ischemic or infarcted area at $37^{\circ} \mathrm{C}$ for $15 \mathrm{~min}$. The parts stained blue were designated as the ischemic area, while those parts without blue staining were designated as the infarct area. Finally, the different parts of the ventricle were weighed respectively. Myocardial AAR was indicated with the percentage of the left ventricle. Infarct size was demonstrated as the percentage of the AAR (12).

Terminal deoxynucleotidyl transferase dUTP nick end labeling (TUNEL) assay. Myocardial apoptosis was analyzed with the TUNEL technique (Roche Diagnostics $\mathrm{GmbH}$, Mannheim, Germany) according to the manufacturer's instructions. Nuclei were labeled with hematoxylin and the number of TUNEL-positive cells were observed using light microscopy and calculated by randomly counting in 10 fields in the section and were demonstrated with percentage of normal nuclei.

Enzyme-linked immunosorbent assay (ELISA). The cell culture supernatants were collected, and the concentration of creatine kinase-MB isoenzyme (CK-MB; CSB-E14403r; Cusabio Biotech Co., Ltd., College Park, MD, USA), IL-6 (Invitrogen; Thermo Fisher Scientific, Inc.) and TNF- $\alpha$ 




Figure 1. Inhibition of the Rho-kinase signal pathway diminishes PARP expression in I/R-inducing injury. (A) Diagram of the experimental protocol in the current study. (B) Western blot analysis of the protein levels of p-MYPT-1 and PARP in different groups of H9C2 cells (Sham, DMSO + I/R, Y-27632 + I/R and $3-\mathrm{AB}+\mathrm{I} / \mathrm{R})$. (C) Relative quantitative analysis of p-MYPT-1 and PARP expression. ${ }^{* *} \mathrm{P}<0.01$ vs. Sham; ${ }^{\#} \mathrm{P}<0.05$ vs. DMSO $+\mathrm{I} / \mathrm{R}$; NS vs. DMSO $+\mathrm{I} / \mathrm{R}$. Similar observations were obtained in three independent experiments. PARP, poly adenosine diphosphate-ribose polymerase; I/R, ischemia/reperfusion; p-, phosphorylated; MYPT-1, myosin phosphatase target subunit 1; DMSO, dimethyl sulfoxide; 3-AB, 3-aminobenzamide; NS, nonsignificant.

(Invitrogen; Thermo Fisher Scientific, Inc.) were measured using a commercially available ELISA kit in accordance with the manufacturer's instructions.

Assay of $\mathrm{LDH}$ and $\mathrm{NO}$ release. Myocardial damage was examined by measuring lactate dehydrogenase (LDH) activity in culture media with the LDH-Cytotoxicity Assay Kit (C0016; Beyotime Institute of Biotechnology, Haimen, China) according to the manufacturer's recommendations. NO in the serum of rats was measured indirectly using the Griess reagent kit (Beyotime Institute of Biotechnology) according to the manufacturer's recommendations. The optical density was detected at $490 \mathrm{~nm}$ (for LDH) or $540 \mathrm{~nm}$ (for NO) using the Multiskan GO microplate reader (Thermo Fisher Scientific, Inc.). The LDH activity and NO concentration were determined using a standard curve.

Measurement of $\mathrm{NAD}^{+}$levels. Left ventricles were washed with cold phosphate-buffered saline and were ground into a fine powder with a pestle and mortar in liquid nitrogen. The powder was transferred into $1.5 \mathrm{ml}$ Eppendorf tube and added $400 \mu \mathrm{l}$ nicotinamide adenine dinucleotide phosphate (NADP)/NADPH extraction buffer to homogenize. The samples were centrifuged at $16,000 \mathrm{x} \mathrm{g}$ for $5 \mathrm{~min}$ at $4^{\circ} \mathrm{C}$. Subsequently, the extracted samples were transferred into new tubes. Samples were heated to $60^{\circ} \mathrm{C}$ for $30 \mathrm{~min}$ in a water bath and then were cooled on ice. Following this, $50 \mu \mathrm{l}$ of the above solution was added into a 96 -well bottom assay plate in duplicate, NADP cycling mix (K337-100; BioVision, Inc., Milpitas, CA, USA) was added to each well, and the plate was incubated at room temperature for $5 \mathrm{~min}$. Finally, $10 \mu \mathrm{l}$ developer was added and the samples were incubated for $2 \mathrm{~h}$. The absorbance was measured at $450 \mathrm{~nm}$.

Statistical analysis. All data was presented as a result of three or four independent experiments. All data were expressed as the mean \pm standard deviation, and was analyzed via one-way analysis of variance and two-tailed Student's t-test. In all cases, values of $\mathrm{P}<0.05$ were considered to indicate a statistically significant difference.

\section{Results}

Inhibition of the ROCK signaling pathway diminishes PARP expression in $I / R$-induced injury. To identify the role of ROCK and PARP in the myocardial I/R injury, the activation of ROCK and the expression of PARP by were analyzed by western blotting. As presented in Fig. 1B and C, compared with the sham control, a significant increase in expression of p-MYPT-1, a well-known ROCK specific substrate, and PARP were detected during I/R. At the same time, in order to confirm the associations of ROCK and PARP, the ROCK and PARP specific inhibitors Y-27632 and 3-AB were used in I/R. As presented in Fig. 1B and C, the expression levels of p-MYPT-1 and PARP were significantly decreased in the presence of the two inhibitors, respectively. Notably, the expression of PARP was additionally abrogated by the ROCK inhibitor Y-27632. Taken together, these data indicate that ROCK and PARP were upregulated and ROCK may be an upstream signaling molecule of PARP in myocardial I/R injury.

ROCK-mediated regulation of PARP expression promotes myocardial cell apoptosis in I/R-induced injury. To further investigate the effect of ROCK and PARP for myocardial cell function, the I/R-induced cell apoptosis was detected with Hoechst staining assay. As presented in Fig. 2A and B, the percentage of apoptotic cells in the DMSO plus I/R group was significantly increased compared with that of the sham group. The nuclear edge of the sham control group was clear and integrated. Nevertheless, apoptotic salient features including chromatin condensation and DNA fragmentation etc. were identified in the DMSO plus I/R group. The percentage of 

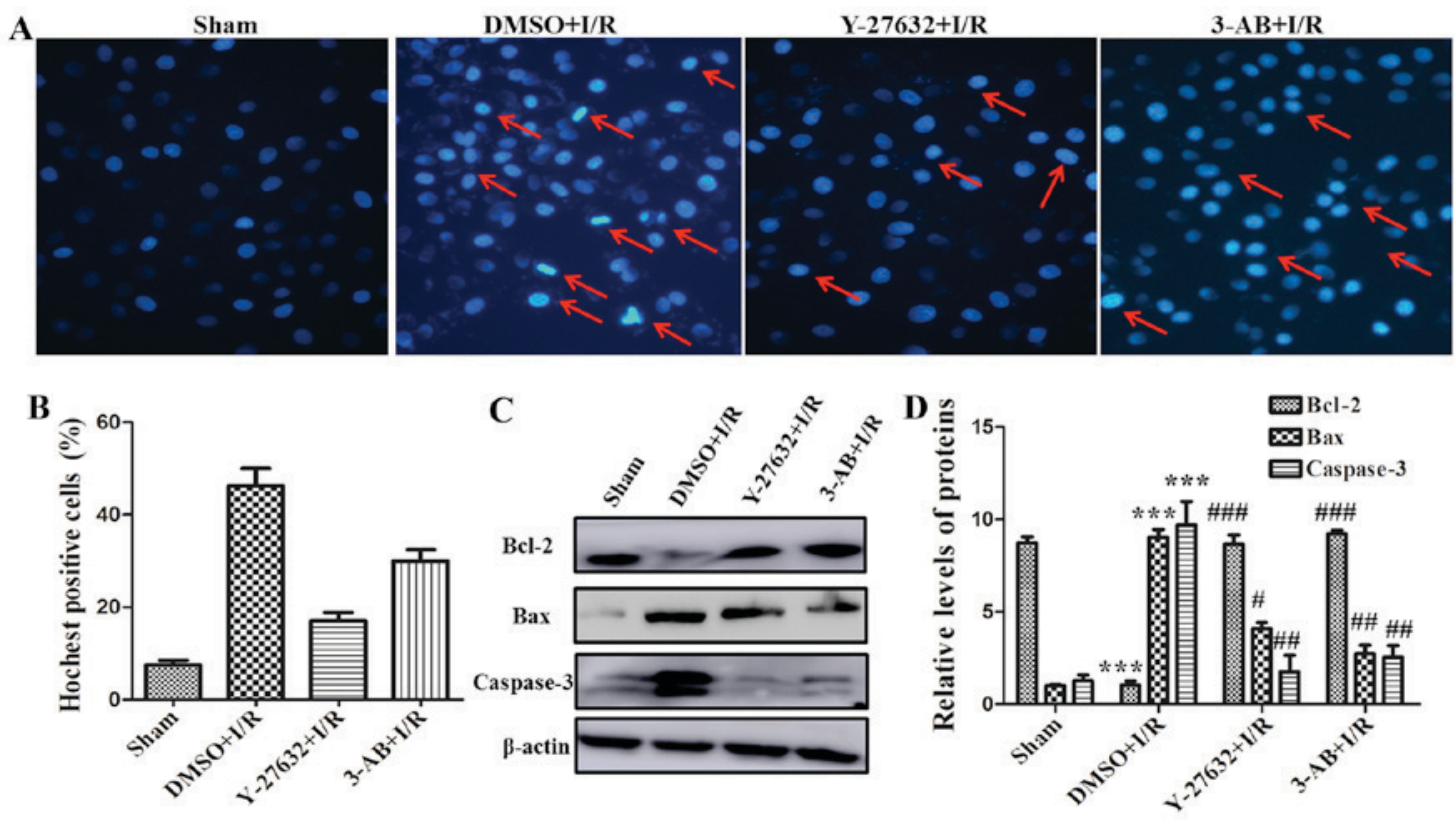

Figure 2. ROCK regulating PARP expression promotes myocardial cell apoptosis in I/R-inducing injury. (A) Hoechst staining was completed (magnification, x200; red arrows, chromatin condensation, DNA fragmentation and chromatin margination). (B) The histogram present the rates of Hoechst-positive cells. (C) The expression levels of Bcl-2, Bax and caspase-3 were measured in different groups of H9C2 cells by western blotting. (D) Relative quantitative analysis of Bcl-2, Bax and caspase-3 expression. ${ }^{* * *} \mathrm{P}<0.001$ vs. Sham; ${ }^{\#} \mathrm{P}<0.05,{ }^{\# \#} \mathrm{P}<0.01$ and ${ }^{\# \# \#} \mathrm{P}<0.001$ vs. DMSO + I/R; ${ }^{\mathrm{P}}<0.05$, vs. Y-27632 + I/R. Similar observations were obtained in three independent experiments. ROCK, Rho-kinase; PARP, poly adenosine diphosphate-ribose polymerase; I/R, ischemia/reperfusion; Bcl-2, B-cell lymphoma 2; Bax, Bcl-2-associated X protein; DMSO, dimethyl sulfoxide.

apoptotic cells was significantly decreased in the presence of Y-27632 or 3-AB. The number of apoptotic cells in the Y-27632 inhibitor group was relatively less compared with the $3-\mathrm{AB}$ group. In addition, the expression of the apoptosis-associated molecules, including Bcl-2, Bax and caspase- 3 were assessed by western blot analysis. As presented in Fig. $2 \mathrm{C}$ and $\mathrm{D}$, the expression of apoptosis-promoting proteins, including Bax and caspase-3, were markedly increased in the DMSO plus $\mathrm{I} / \mathrm{R}$ group compared with the sham group. In contrast, the expression of the anti-apoptotic protein $\mathrm{Bcl}-2$ was markedly decreased in DMSO plus I/R group. Otherwise, pretreatment of Y-27632 or 3-AB can significantly increase Bcl-2 expression and reduce Bax and caspase- 3 expression. Taken together, these results indicated that ROCK and PARP served an important role in I/R-induced myocardial cell apoptosis and the effect of Y-27632, a ROCK inhibitor, in reversing cell apoptosis was more significant than in the 3-AB group.

ROCK and PARP inhibition during I/R attenuated myocardial cell apoptosis and infarct size in vivo. In order to evaluate the effect of ROCK and PARP inhibition in vivo, DMSO or corresponding inhibitors were pre-injected and then established as rat models of I/R. As presented in Fig. $3 \mathrm{~A}$ and $\mathrm{B}$, the AAR and infarct size of the hearts were $57.67 \pm 2.52$ and $60.67 \pm 2.52 \%$, respectively in the I/R plus DMSO group. Administration of Y-27632 or 3-AB caused significant reduction of AAR and infarct size. The AAR and infarct size of the hearts were $29.67 \pm 4.73$ and $30.02 \pm 4.10 \%$, respectively in the I/R plus Y-27632 group. The AAR and infarct sizes of the hearts were $44.67 \pm 7.57$ and $46.33 \pm 11.50 \%$, respectively in the I/R plus $3-\mathrm{AB}$ group. In addition, it was observed that the AAR and infarct sizes of the heart in the I/R plus Y-27632 group were always lower than in the I/R plus 3-AB group. Furthermore, myocardial apoptosis was observed with the TUNEL assay. As presented in Fig. 3F and G, TUNEL-positive cells were significantly increased in I/R $(37 \pm 6.24 \%)$. However, the numbers were markedly decreased to $19 \pm 2.08$ and $32 \pm 3.61 \%$ in the presence of the ROCK and PARP inhibitor, respectively. In addition, the content of CK-MB, LDH and NO were measured in the serum. As presented in Fig. 3C-E, it was identified that the content of CK-MB and LDH was significantly enhanced and NO was reduced in the I/R plus DMSO group. Y-27632 or 3-AB were able to markedly downregulate the content of CK-MB and LDH and rescued NO in rat serum, and the effect of Y-27632 inhibition was superior to that of 3-AB. Taken together, these results indicate that inhibition of ROCK activity and PARP reduces myocardial infarct size and cell apoptosis in I/R injury. In summary, the effects of Y-27632 were superior to $3-\mathrm{AB}$.

ROCK/PARP/ERK signaling pathway served a vital role in $I / R$-inducing injury. In order to analyze the unique role of PARP during I/R-inducing injury, the NAD ${ }^{+}$level in the left ventricle was measured. As presented in Fig. 4A, the level of $\mathrm{NAD}^{+}$was reduced in the $\mathrm{I} / \mathrm{R}$ group compared with the sham group. However, Y-27632 or 3-AB significantly augmented the level of $\mathrm{NAD}^{+}$and the effect of Y-27632 was more marked. In addition, as presented in Fig. 4B and C, the level of TNF- $\alpha$ and IL- 6 in rat serum was observed and it was identified that the content of the two cytokines were also increased in I/R. Pre-treatment with Y-27632 or 3-AB decreased their levels in rat serum. In addition, the phosphorylation levels of ERK were measured using western blot analysis. As presented in Fig. 4D and E, it was demonstrated that phosphorylation of 

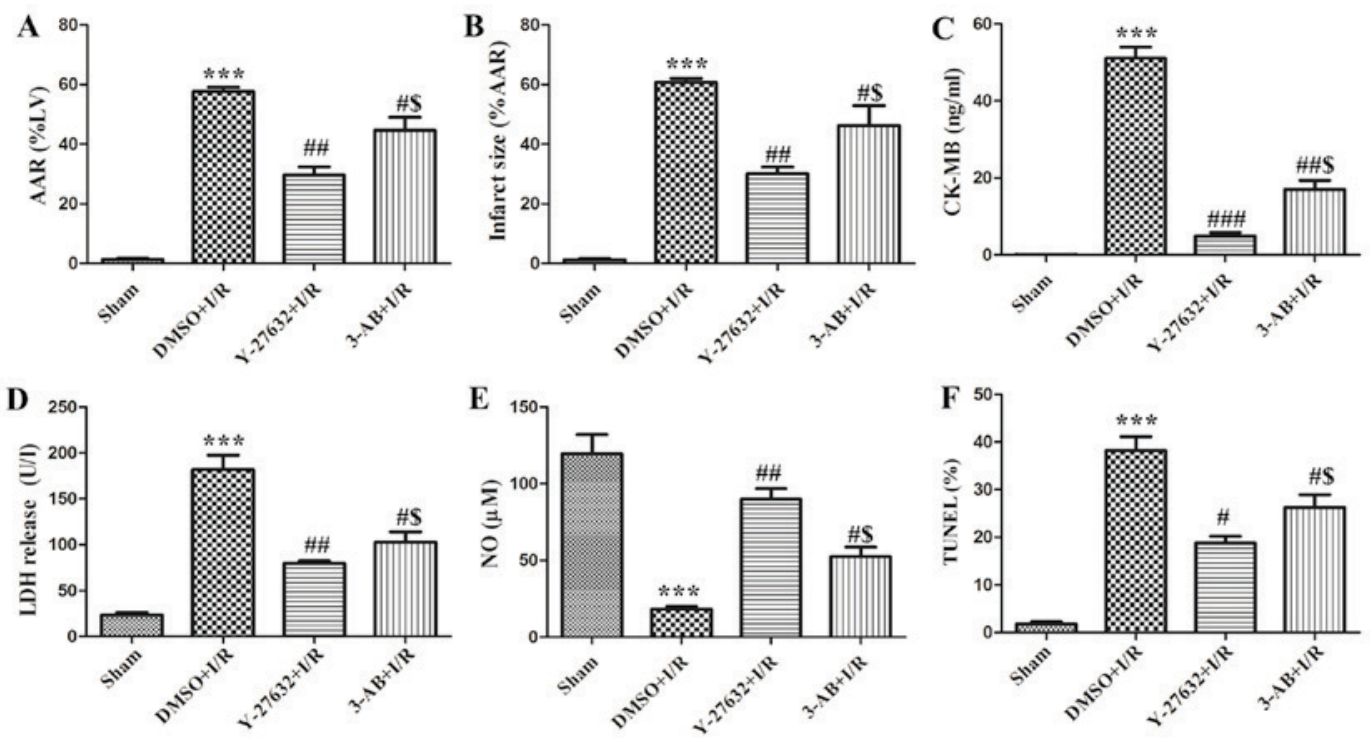

G
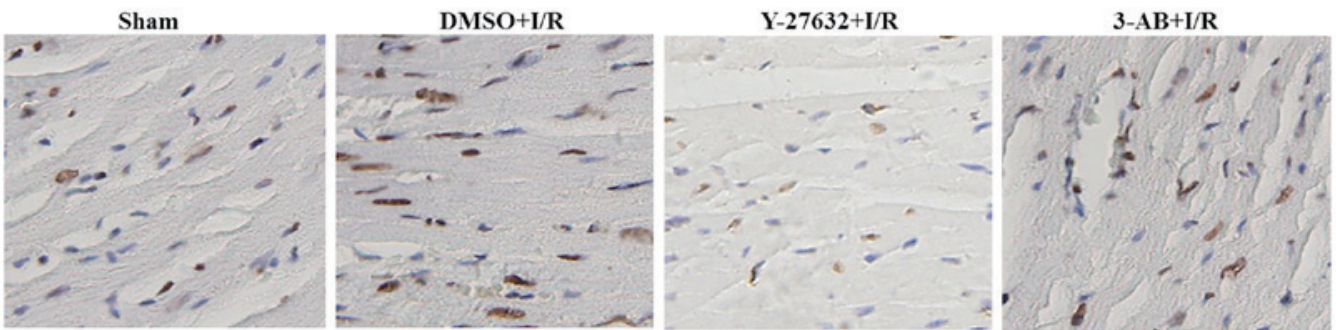

Figure 3. ROCK and PARP inhibition during $\mathrm{I} / \mathrm{R}$ attenuated myocardial cell apoptosis and infarct size in vivo. Changes in (A) myocardial AAR and (B) infarct size of different groups (Sham, DMSO + I/R, Y-27632 + I/R, 3-AB + I/R) in the rat hearts. The content of (C) CK-MB, (D) LDH release and (E) NO levels were detected in the serum of rats. (G) Representative photomicrographs of ventricular tissue stained for TUNEL for DNA breaks in different groups. (F) The histogram shows the percentage of TUNEL positive cells. $n=15 ;{ }^{* * * *} \mathrm{P}<0.001$ vs. Sham; ${ }^{*} \mathrm{P}<0.05,{ }^{\# \#} \mathrm{P}<0.01$ and ${ }^{\# \# \#} \mathrm{P}<0.001$ vs. DMSO + I/R; ${ }^{\mathrm{S}} \mathrm{P}<0.05$, vs. Y-27632 + I/R. ROCK, Rho-kinase; PARP, poly adenosine diphosphate-ribose polymerase; I/R, ischemia/reperfusion; DMSO, dimethyl sulfoxide; 3-AB, 3-aminobenzamide; TUNEL, terminal deoxynucleotidyl transferase dUTP nick end labeling; AAR, area at risk; CK-MB, creatine kinase-MB; LDH, lactate dehydrogenase; $\mathrm{NO}$, nitric oxide.
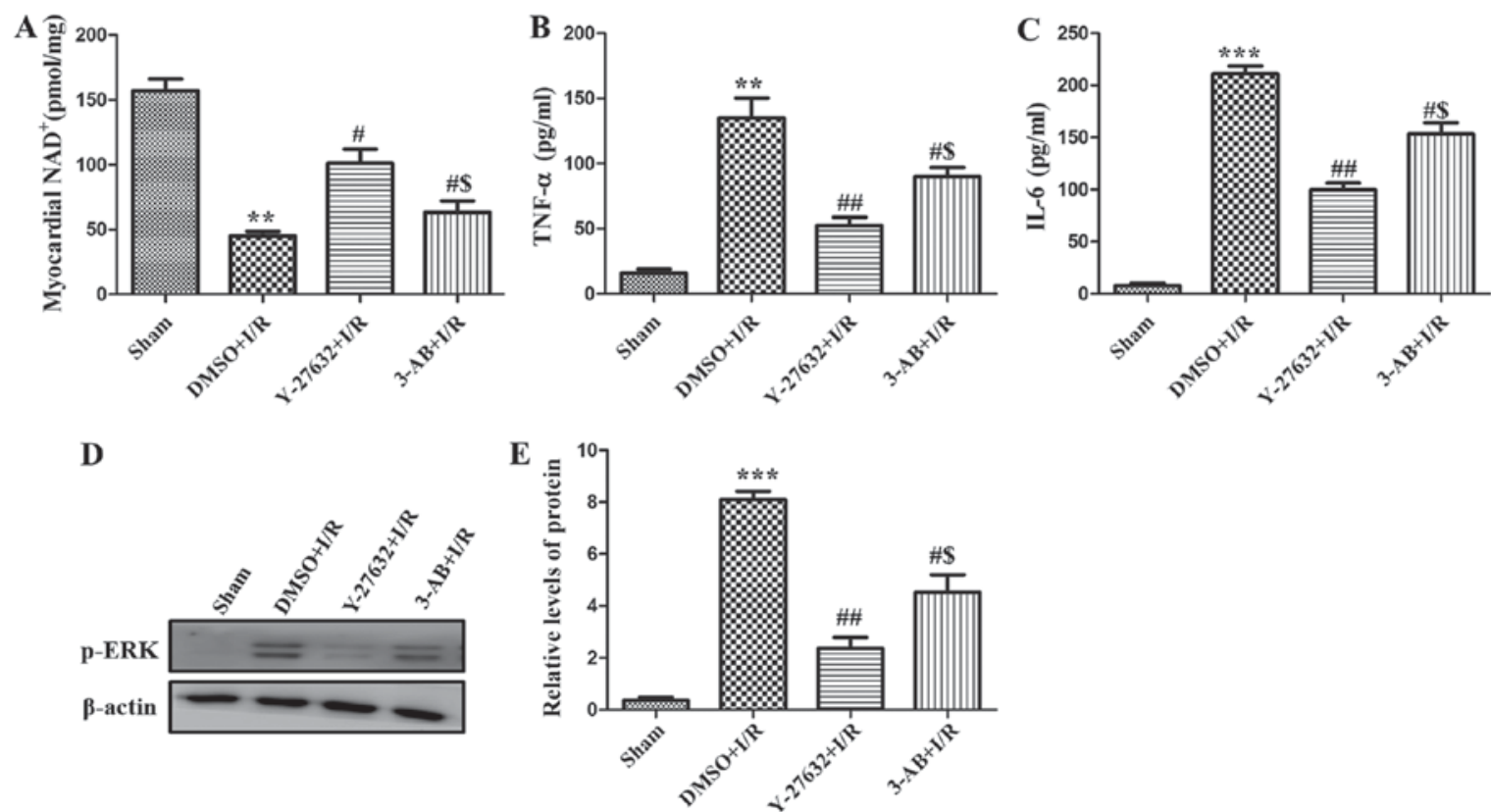

Figure 4. ERK signal pathway is an important in I/R-inducing injury. (A) NAD level in the left ventricle were measured. (B) TNF- $\alpha$ and (C) IL-6 in the serum of rats were determined by ELISA. (D) Western blot analysis of protein level of p-ERK in different groups. (E) Relative quantitative analysis of p-ERK expression. ${ }^{* *} \mathrm{P}<0.01$ and ${ }^{* * *} \mathrm{P}<0.001$ vs. Sham; ${ }^{*} \mathrm{P}<0.05$ and ${ }^{\# \#} \mathrm{P}<0.01$ vs. DMSO + I/R; ${ }^{{ }^{*}} \mathrm{P}<0.05$ vs. $\mathrm{Y}-27632+\mathrm{I} / \mathrm{R}$. Similar observations were obtained in three independent experiments. ERK, extracellular signal-related kinase; I/R, ischemia/reperfusion; NAD, nicotinamide adenine dinucleotide; TNF- $\alpha$, tumor necrosis factor $\alpha$; IL-6, interleukin 6; p-, phosphorylated; DMSO, dimethyl sulfoxide; 3-AB, 3-aminobenzamide. 
ERK was clearly increased in the hearts of the I/R group and decreased in the pre-treatment of Y-27632 or 3-AB groups. The expression of p-ERK in the Y-27632 group was lower than that of the 3-AB group. Taken together, these data indicate that the MAPK/ERK signaling pathway serves an important role in the regulation of I/R injury by ROCK and PARP.

\section{Discussion}

In the current study, the activation of ROCK and the expression of PARP were demonstrated to be increased in the hypoxia-reoxygenation injury model in the $\mathrm{H} 9 \mathrm{C} 2$ cell line, and they were observed to aggravate the apoptosis of myocardial cells. In addition, it was identified that there was an association between ROCK and PARP via a corresponding inhibitors pretreatment assay. PARP may be located downstream of ROCK and regulated myocardial cell apoptosis in heart I/R injury. Furthermore, it was validated that the MAPK/ERK signaling pathway served a prominent role in heart $\mathrm{I} / \mathrm{R}$ injury by effecting production of proinflammatory cytokines, including TNF- $\alpha$ and IL- 6 . The results of the present study suggest that ROCK increases myocardial apoptosis via the PARP/ERK signaling pathway.

Ischemic heart disease has become one of the leading factors of death worldwide (14). Protection of the heart against injury in $\mathrm{I} / \mathrm{R}$ injury remains a challenge, thus it is particularly important to clarify the pathogenesis and role of important molecules during I/R. ROCK is a major regulator of the execution phase of apoptosis, including cell contraction, dynamic membrane blebbing, nuclear disintegration and fragmentation of apoptotic cells into apoptotic bodies $(15,16)$. Specifically, when DNA strand-breaks happen, PARP, a key protein participating in DNA repair (17), binds rapidly to the DNA strand-breaks and converts NAD into long branched polymers to attach onto a variety of nuclear proteins $(12,18)$. Nevertheless, the activation of excessive PARP may cause a negative effect via a caspase-independent pathway-mediated cell apoptosis (19). Previously, although it has been reported that ROCK and PARP are involved in I/R injury and they aggravate cell apoptosis and augment infarct size $(8,9,12)$, the correlation and functional mechanism of ROCK and PARP remain to be fully understood. In the present study, ROCK was demonstrated to be located upstream of PARP to induce myocardial apoptosis.

Previous studies have investigated the function of the the MAPK signaling pathways in I/R, including ERK, c-Jun N-terminal kinase (JNK) and p38 pathways. For example, Yang et al (20) identified that CRGP effectively improved I/R injury of the brain tissue through a reduction in JNK and p38 phosphorylation and an increase in ERK phosphorylation in the MAPK pathway. Zhang et al (9) demonstrated that ROCK enhanced cardiomyocyte apoptosis in the heart I/R via promotion of JNK-mediated apoptosis-inducing factor translocation. Nevertheless, it was demonstrated in the current study that inhibition of ROCK and PARP can reduce the phosphorylation of ERK and the resulting production of proinflammatory factors.

In conclusion, the present study elucidated a novel pathway for ROCK and PARP in the regulation of heart I/R injury. ROCK promotes the expression of PARP to accelerate myocardial apoptosis through raising ERK phosphorylation and the release of TNF- $\alpha$ and IL-6. Given the pathological role of ROCK and PARP in I/R injury, ROCK/PARP/ERK may aid in the understanding of the mechanism of myocardial cell apoptosis and provide novel suggestions for the therapy of I/R.

\section{Acknowledgements}

The present study was supported in part by grants from the Promotive Research Fund for Excellent Young and Middle-aged Scientists of Shandong Province (grant no. BS2014YY037), the Project Funded by China Postdoctoral Science Foundation (grant no. 2013M541926), the Postdoctoral Innovation Project Special Foundation of Shandong Province (grant no. 201302031) and the Science and Technology Development Plans of Shandong Province (grant no. 2012GSF21807).

\section{References}

1. Peuhkurinen K: Myocardial reperfusion-a double-edged sword? Duodecim 105: 822-830, 1989 (In Finnish).

2. Kurian GA, Rajagopal R, Vedantham S and Rajesh M: The role of oxidative stress in myocardial ischemia and reperfusion injury and remodeling: Revisited. Oxid Med Cell Longev 2016: 1656450, 2016

3. Patil KD, Halperin HR and Becker LB: Cardiac arrest: Resuscitation and reperfusion. Circ Res 116: 2041-2049, 2015.

4. Matsushima S, Tsutsui H and Sadoshima J: Physiological and pathological functions of NADPH oxidases during myocardial ischemia-reperfusion. Trends Cardiovasc Med 24: 202-205, 2014.

5. Julian L and Olson MF: Rho-associated coiled-coil containing kinases (ROCK): Structure, regulation, and functions. Small GTPases 5: e29846, 2014

6. Minambres R, Guasch RM, Perez-Aragó A and Guerri C: The RhoA/ROCK-I/MLC pathway is involved in the ethanol-induced apoptosis by anoikis in astrocytes. J Cell Sci 119: 271-282, 2006.

7. Chang J, Xie M, Shah VR, Schneider MD, Entman ML, Wei L and Schwartz RJ: Activation of Rho-associated coiled-coil protein kinase 1 (ROCK-1) by caspase-3 cleavage plays an essential role in cardiac myocyte apoptosis. Proc Natl Acad Sci USA 103: 14495-14500, 2006.

8. Zhang J, Bian HJ, Li XX, Liu XB, Sun JP, Li N, Zhang Y and Ji XP: ERK-MAPK signaling opposes rho-kinase to reduce cardiomyocyte apoptosis in heart ischemic preconditioning. Mol Med 16: 307-315, 2010.

9. Zhang J, Li XX, Bian HJ, Liu XB, Ji XP and Zhang Y: Inhibition of the activity of Rho-kinase reduces cardiomyocyte apoptosis in heart ischemia/reperfusion via suppressing JNK-mediated AIF translocation. Clin Chim Acta 401: 76-80, 2009.

10. Abe K, Shimokawa H, Morikawa K, Uwatoku T, Oi K, Matsumoto Y, Hattori T, Nakashima Y, Kaibuchi K, Sueishi K and Takeshit A: Long-term treatment with a Rho-kinase inhibitor improves monocrotaline-induced fatal pulmonary hypertension in rats. Circ Res 94: 385-393, 2004.

11. Harris JL, Jakob B, Taucher-Scholz G, Dianov GL, Becherel OJ and Lavin MF: Aprataxin, poly-ADP ribose polymerase 1 (PARP-1) and apurinic endonuclease 1 (APE1) function together to protect the genome against oxidative damage. Hum Mol Genet 18: 4102-4117, 2009.

12. Song ZF, Ji XP, Li XX, Wang SJ, Wang SH and Zhang Y: Inhibition of the activity of poly (ADP-ribose) polymerase reduces heart ischaemia/reperfusion injury via suppressing JNK-mediated AIF translocation. J Cell Mol Med 12: 1220-1228, 2008.

13. Li B, Li R, Zhang C, Bian HJ, Wang F, Xiao J, Liu SW, Yi W, Zhang MX, Wang SX, et al: MicroRNA-7a/b protects against cardiac myocyte injury in ischemia/reperfusion by targeting poly (ADP-ribose) polymerase. PLoS One 9: e90096, 2014. 
14. Bolli R, Becker L, Gross G, Mentzer R Jr, Balshaw D and Lathrop DA; NHLBI Working Group on the Translation of Therapies for Protecting the Heart from Ischemia: Myocardial protection at a cross roads: The need for translation into clinical therapy. Circ Res 95: 125-134, 2004.

15. Erwig LP, McPhilips KA, Wynes MW, Ivetic A, Ridley AJ and Henson PM: Differential regulation of phagosome maturation in macrophages and dendritic cells mediated by Rho GTPases and ezrin-radixin-moesin (ERM) proteins. Proc Natl Acad Sci USA 103: 12825-12830, 2006.

16. Tosello-Trampont AC, Nakada-Tsukui $\mathrm{K}$ and Ravichandran KS: Engulfment of apoptotic cells is negatively regulated by Rho-mediated signaling. J Biol Chem 278: 49911-49919, 2003.

17. Meng X, Song W, Deng B, Xing Z and Zhang W: 3-aminobenzamide, one of poly(ADP-ribose)polymerase-1 inhibitors, rescues apoptosis in rat models of spinal cord injury. Int J Clin Exp Pathol 8: 12207-12215, 2015.
18. Wang SJ, Wang SH, Song ZF, Liu XW, Wang R and Chi ZF: Poly(ADP-ribose) polymerase inhibitor is neuroprotective in epileptic rat via apoptosis-inducing factor and Akt signaling. Neuroreport 18: 1285-1289, 2007.

19. Meli E, Pangallo M, Picca R, Baronti R, Moroni F and Pellegrini-Giampietro DE: Differential role of poly(ADP-ribose) polymerase-1 in apoptotic and necrotic neuronal death induced by mild or intense NMDA exposure in vitro. Mol Cell Neurosci 25: 172-180, 2004.

20. Yang SI, Yuan Y, Jiao S, Luo QI and Yu J: Calcitonin gene-related peptide protects rats from cerebral ischemia/reperfusion injury via a mechanism of action in the MAPK pathway. Biomed Rep 4: 699-703, 2016 